\title{
Atomically Precise Alkynyl-Protected Metal Nanoclusters as a Model Catalyst: Observation of Promoting Effect of Surface Ligands on Catalysis by Metal Nanoparticles
}

\author{
Yu Wang, ${ }^{1}$ Xian-Kai Wan, ${ }^{1}$ Liting Ren, ${ }^{1}$ Haifeng Su, ${ }^{1}$ Gang $\mathrm{Li},{ }^{2}$ Sami Malola, ${ }^{3}$ \\ Shuichao Lin, ${ }^{1}$ Zichao Tang, ${ }^{1,2}$ Hannu Häkkinen, ${ }^{3}$ Boon K Teo, ${ }^{1}$ Quan-Ming Wang, ${ }^{1 *}$ \\ Nanfeng Zheng ${ }^{1 *}$ \\ ${ }^{1}$ Collaborative Innovation Center of Chemistry for Energy Materials, State Key Laboratory \\ for Physical Chemistry of Solid Surfaces, and Department of Chemistry, College of \\ Chemistry and Chemical Engineering, Xiamen University, Xiamen 361005, China \\ ${ }^{2}$ State Key Laboratory of Molecular Reaction Dynamics, Dalian Institute of Chemical \\ Physics, Chinese Academy of Sciences, Dalian 116023, China \\ ${ }^{3}$ Departments of Physics and Chemistry, Nanoscience Center, University of Jyväskylä, \\ FI-40014 Jyväskylä, Finland
}

\section{Experimental Details}

Reagents: Silver acetate (AgOAc, purity 98\%), borane-tert-butylamine complex $\left[\left(\mathrm{CH}_{3}\right)_{3} \mathrm{CNH}_{2} \cdot \mathrm{BH}_{3}\right.$, purity $\left.97 \%\right]$, phenylacetylene $(\mathrm{PhC} \equiv \mathrm{CH}$, purity $98 \%)$, tertbutylacetylene $\left(\mathrm{C}_{6} \mathrm{H}_{9} \mathrm{C} \equiv \mathrm{CH}\right.$, purity $\left.98 \%\right)$ were purchased from Alfa Aesar Chemical Reagent Co. Ltd. (Tianjin, China), Sodium methoxide $\left(\mathrm{CH}_{3} \mathrm{ONa}\right.$, powder, purity $\left.99 \%\right)$ was purchased from J\&K Scientific Ltd. (Bejing, China), chloroform ( $\mathrm{CHCl}_{3}$, A.R.), methanol $\left(\mathrm{CH}_{3} \mathrm{OH}\right.$, A.R.) were purchased from Sinopharm Chemical Reagent Co. Ltd. (Shanghai, China). The water used in all experiments was ultrapure. All reagents were used as received without further purification. $\mathrm{AuSMe}_{2} \mathrm{Cl}$ and $\mathrm{PhC} \equiv \mathrm{CAuPPh}_{3}$ were prepared according to literature methods. ${ }^{1,2}$

Synthesis of $\mathbf{A u}_{34} \mathbf{A g}_{28}(\mathbf{P A})_{34}$. 6mg $\mathrm{AuSMe}_{2} \mathrm{Cl}$ was first dissolved in the mixture solution of chloroform and methanol. 2.2 $\mu \mathrm{L}$ phenylacetylene was added to a $\mathrm{MeONa} / \mathrm{MeOH}$ solution $(1 \mathrm{mg} / \mathrm{mL})$. The solutions were mixed and stirred for $10 \mathrm{~min}$, then $5 \mathrm{mg} \mathrm{AgCH}_{3} \mathrm{COO}$ was added and stirred for 5min. The reducing agent, tert-butylamineborane $(3.6 \mathrm{mg})$, was added to the mixture under vigorous stirring. The reaction was aged for $12 \mathrm{~h}$ at room temperature. The solution was centrifuged for 4 $\mathrm{min}$ at $14000 \mathrm{r} / \mathrm{min}$. The brown supernatant was subjected to natural evaporation in the dark. Black block crystals were obtained in a yield of $\sim 28 \%$ (based on $\mathrm{Au}$ ).

The cluster was also readily prepared by chemically reducing the mixture of $\mathrm{PhC} \equiv \mathrm{CAuPPh}_{3}, \mathrm{PhC} \equiv \mathrm{CAu}$ and $\mathrm{PhC} \equiv \mathrm{CAg}$ metal precursors by $\mathrm{NaBH}_{4}$. In a typical synthesis, to $4.0 \mathrm{~mL} \mathrm{CH}_{2} \mathrm{Cl}_{2}$ suspension containing $\mathrm{PhC} \equiv \mathrm{CAuPPh}_{3}(9.3 \mathrm{mg}, 0.0167$ 
mmol), $\mathrm{PhC} \equiv \mathrm{CAu}(29.8 \mathrm{mg}, 0.1 \mathrm{mmol})$ and $\mathrm{PhC} \equiv \mathrm{CAg}$ (20.8 mg, 0. 1mmol), a freshly prepared solution of $\mathrm{NaBH}_{4}(0.82 \mathrm{mg}$ in $1.0 \mathrm{~mL}$ of ethanol $)$ was added dropwise under vigorous stirring. The reaction continued for $24 \mathrm{~h}$ at room temperature in the dark, and a small amount of black solid appeared at the flask bottom. The mixture was evaporated to dryness to give a dark solid, which was extracted with $\mathrm{CH}_{2} \mathrm{Cl}_{2}(3 \times 2.0$ $\mathrm{mL})$. After centrifuged for $4 \mathrm{~min}$ at $10000 \mathrm{r} / \mathrm{min}$, the volume of the supernatunt was reduced to $2.0 \mathrm{~mL}$, which was subject to diffusion of $\mathrm{n}$-hexane to give black plate crystals of $\mathrm{Au}_{34} \mathrm{Ag}_{28}(\mathrm{PhC} \equiv \mathrm{C})_{34}$ after two weeks (5.2 mg, 13\% based on $\mathrm{Au}$ ).

Preparation of $\mathrm{TiO}_{2}(\mathbf{P 2 5})$-supprted Au nanoparticles. A $100 \mathrm{~mL}$ solution of $\mathrm{HAuCl}_{4} \cdot 4 \mathrm{H}_{2} \mathrm{O}(1 \mathrm{mg} / \mathrm{mL})$ was heated to $70^{\circ} \mathrm{C}$ under vigorous stirring. After adjusting the $\mathrm{pH}$ to 7 using $\mathrm{NaOH}$ solution, $1 \mathrm{~g} \mathrm{TiO}_{2}$ was added, and the suspension was aged at $70^{\circ} \mathrm{C}$ for $1 \mathrm{hr}$. The solid was collected via centrifugation and washed with deionized water for five times.

Post-treatment of thermally treated catalysts with deprotonated terminal alkynes. The catalyst was dispersed into methanol, then $100 \mathrm{mg} \mathrm{MeONa}$ and $0.2 \mathrm{~mL}$ phenylacetylene / tert- butylacetylene were added. The suspension was stirred at $50^{\circ} \mathrm{C}$ overnight. The solid was collected via centrifugation and washed with methanol for several times.

Physical measurements. UV-Vis absorption spectra were recordedon a Varian Carry 5000 spectrophotometer. Mass spectrum was recorded on an Agilent Technologies ESI-TOF-MS. MALDI mass spectra were collected on a linear-mode time-of-flight (TOF) mass spectrometer (Bruker) using a nitrogen laser $(337 \mathrm{~nm})$. TEM studies were performed on a TECNAI F-30 transmission electron microscope operating at $300 \mathrm{kV}$.

Single-Crystal Analysis. The diffraction data were collected on an Agilent SuperNova X-Ray single crystal diffractometer using $\mathrm{Cu} \operatorname{K\alpha }(\lambda=1.54184 \AA)$ micro-focus X-ray sources at $100 \mathrm{~K}$. The data were processed using CrysAlisPro. ${ }^{3}$ The structure was solved and refined using Full-matrix least-squares based on $F^{2}$ with program SHELXS-97 and SHELXL-97 (Ref. 4) within OLEX2. ${ }^{5}$ Crystallographic data for $\mathrm{Au}_{34} \mathrm{Ag}_{28}(\mathrm{PA})_{34}$ : monoclinic $P 2_{1} / c, a=36.8939(6) \AA, b=22.0660(5) \AA, c=$ 39.9832(7) $\AA, \alpha=90^{\circ}, \beta=102.2164(18)^{\circ}, \gamma=90^{\circ}, V=31813.2(11) \AA^{3}, Z=4, \mathrm{Cu}$ $\mathrm{K} \alpha, T=100 \mathrm{~K}, 2 \theta=143.398^{\circ} .129151$ reflections were measured, of which 60204 were unique with $R_{\text {int }}=0.0586$. Final $R_{1}=6.04 \%$, w $R_{2}=0.1666$ for 2873 parameters and 41811 reflections with $I>2 \sigma(I)$.

TPD-MS studies of $\mathbf{A u}_{34} \mathbf{A g}_{28}(\mathbf{P A})_{34}$ : The TPD-MS experiment was performed on a home-made TPD-TOF analyzer. $3 \mathrm{mg}$ of $\mathrm{Au}_{34} \mathrm{Ag}_{28}(\mathrm{PA})_{34}$ sample was pyrolyzed in a 
small tube heated by heating coil. A K-type thermocouple was put inside the sample tube and insulated from samples to measure the temperature. The heating coil was powered by a precise electric source and adjusted at interval of $10 \mathrm{mV}$. The temperature of sample tube was ramping from room temperature to $400^{\circ} \mathrm{C}$ smoothly at speed of $5 \mathrm{~K} / \mathrm{min}$ controlled by computer. The desorbed spices were ionized by a UV lamp at position very close to the sample tube with phonon energy of $10.6 \mathrm{eV}$, and then transferred to TOF analyzer by an ion optical system. The TOF analyzer had a resolution of more than 5000, and the sensitivity of ppb level. All those steps were processed in high vacuum of about $3 \times 10^{-5} \mathrm{~Pa}$. The mass spectrum and sample temperature were acquired and recorded every second. Each spectrum is an accumulation of 10000 spectra gathered at interval of $100 \mu \mathrm{s}$.

Catalytic oxidation of triethylsilane: To a mixture of triethylsilane $(2.5 \mathrm{mmol})$, water $(1 \mathrm{~mL})$ and acetone $(5 \mathrm{~mL}), 10 \mathrm{mg}$ catalyst $(1 \mathrm{wt} \%)$ was add. The reaction was proceeding at $50^{\circ} \mathrm{C}$ in air for half an hour. The product was analyzed by GC. The detailed analysis conditions were: oven temperature : $80^{\circ} \mathrm{C}$ for $3 \mathrm{mins}$ and then ramp from $80^{\circ} \mathrm{C}$ to $240^{\circ} \mathrm{C}$ at a rate of $40^{\circ} \mathrm{C} / \mathrm{min}$; injection and detection temperature: $270^{\circ} \mathrm{C}$; 1 -octane as an internal standard.

Computational methods: The calculations were performed with density functional theory as implemented in the code-package GPAW. ${ }^{6}$ GPAW uses the projector augmented wave method in a real-space grid and the setup includes scalar relativistic corrections. We performed the ground state calculation and superatom state analysis directly for the experimentally resolved crystal structure using the PBE functional, neutral total charge and $0.2 \AA$ grid spacing. The analysis of the superatom states was done by projecting the wave functions to spherical harmonics with respect to the center of the mass of the cluster as described in Ref. 7 . We also analyzed separately d-bands and sp-band positions of the Ag- and Au-atoms of the cluster. The optical absorption spectra were calculated using the Casida formalism of the linear response time dependent density functional theory (LR-TDDFT) together with the PBE functional. ${ }^{8}$ The individual oscillator strengths were folded to a continuous absorption spectrum by using a Gaussian width of $0.075 \mathrm{eV}$. The absorption peaks of the calculated spectra were analyzed using the so called transition contribution map (TCM) analysis method that is described in Ref. 9.

Discussion on the structural consequences of the distortion in Shell 1: There are several important structural consequences of the distortion in Shell 1. First, a perfect interpenetrating biicosahedra (ibi) should have 30 triangular faces. If fully capped, it would have produced a second shell of $30 \mathrm{Ag}$ atoms. Ten pairs of them are mirror images with respect to the equatorial plane. Five of the dihedral angles between the 
pairs are convex and the other five are concave. Owing to the equatorial distortion, three of the five concave dihedral angles (corresponding to the three long edges) are significantly smaller than the remaining two $\left(167.679^{\circ}\right.$ vs $\left.175.825^{\circ}\right)$. As a result, each of three former pairs are "capped" with only one Ag atom (hereafter denoted as $A$ atoms), whereas each of the remaining pairs are capped by two Ag atoms. All other triangular faces are each capped by one Ag atom, making a total of 30-3 = 27 Ag atoms in Shell-2. Second, each $A$ atom forms a highly distorted square pyramid with atoms from the Shell-1, two being on the equatorial plane and two off the plane of the first shell. In reality, however, $A$ atoms are 12-coordinated with 4 atoms each from Shell-1(Au), Shell-2 (Ag), and Shell-3 (Au) shells in a 4:4:4 arrangement. The coordination sphere of $A$ atoms can thus be described as a highly distorted cuboctahedron. Third, the equatorial belt of Shell-2, instead of having six edge-sharing hexagons, turns into four pentagons and one square. Together with the other 12 pentagonal faces, Shell-2 has a total of 17 faces. Capping these faces gives rise to the outermost shell, Shell-3, of $\mathrm{Au}_{17}$ coordinated with 34 PA ligands. Fifteen of the 17 monomeric staples $(\mathrm{PhC} \equiv \mathrm{C}-\mathrm{Au}-\mathrm{C} \equiv \mathrm{CPh}$ ) are self-assembled into three pentagonal stripes on the quasi-spherical surface of the $(\mathrm{AuAg})_{45}$ kernel. Each of these stripes is also staggered with respect to one another. The remaining two staples locate on the top and bottom of the kernel. Such a crystallographic evidence that highly ordered patterns of staple motifs can form on nanoclusters has also been observed in the recently reported $\mathrm{Au}_{133}(\mathrm{SR})_{52} .{ }^{\mathrm{S} 10, \mathrm{~S} 11}$ Interestingly, despite the $E D$ and the resulting distortions in Shells 1 and 2, Shell-3 can be likened to an expanded version of Shell-1 with long, nonbonding $\mathrm{Au}$...Au distances. The point-group symmetry of the metal framework is lowered from $D_{5 h}$ to $C_{2 v}$. 


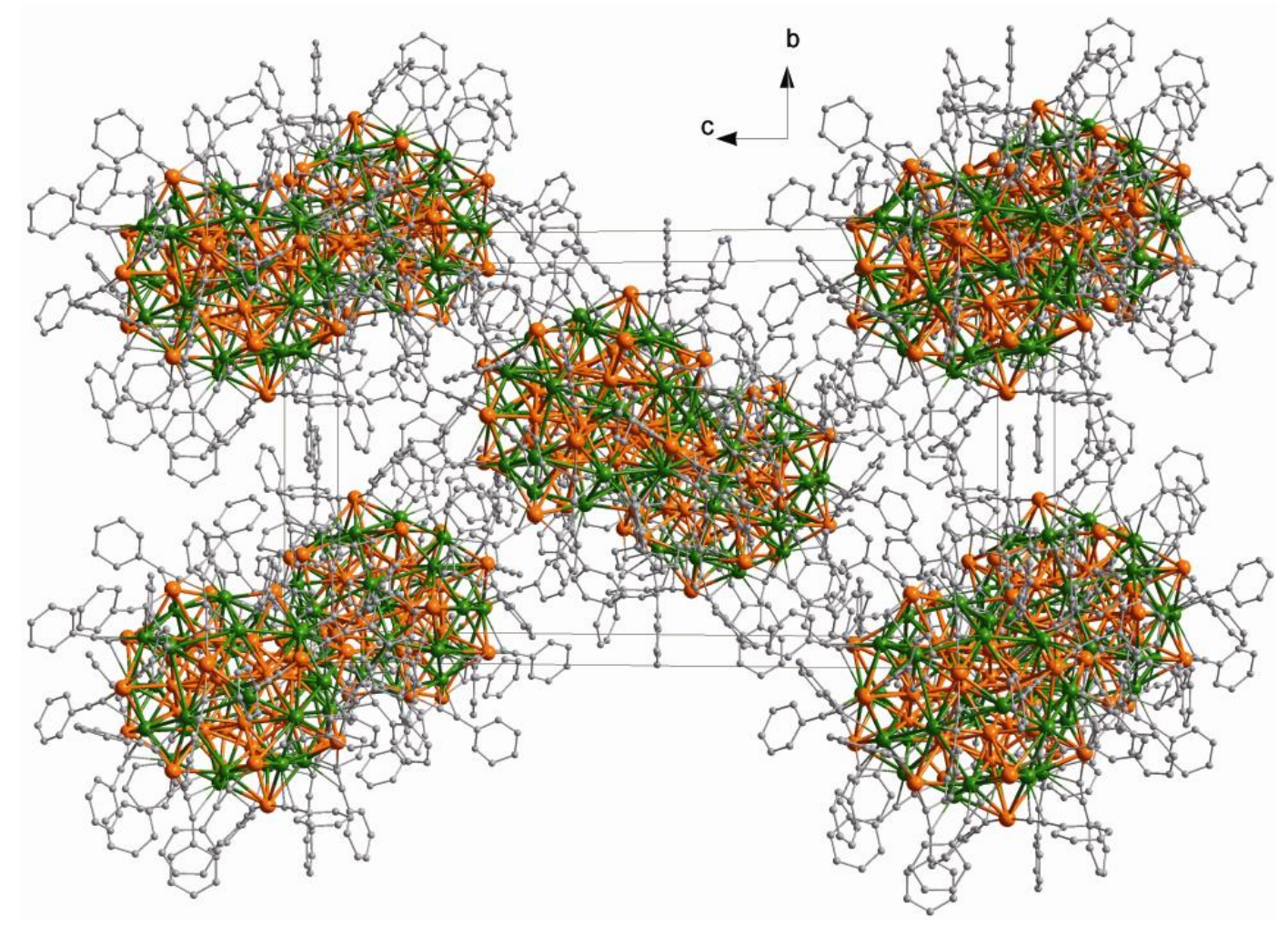

Figure S1. The packing structure of $\mathrm{Au}_{34} \mathrm{Ag}_{28}(\mathrm{PA})_{34}$ nanoclusters in their single crystals. Color legend: gold and purple sphere, $\mathrm{Au}$; green sphere, $\mathrm{Ag}$; grey sphere, $\mathrm{C}$. All hydrogen atoms are omitted for clarity.

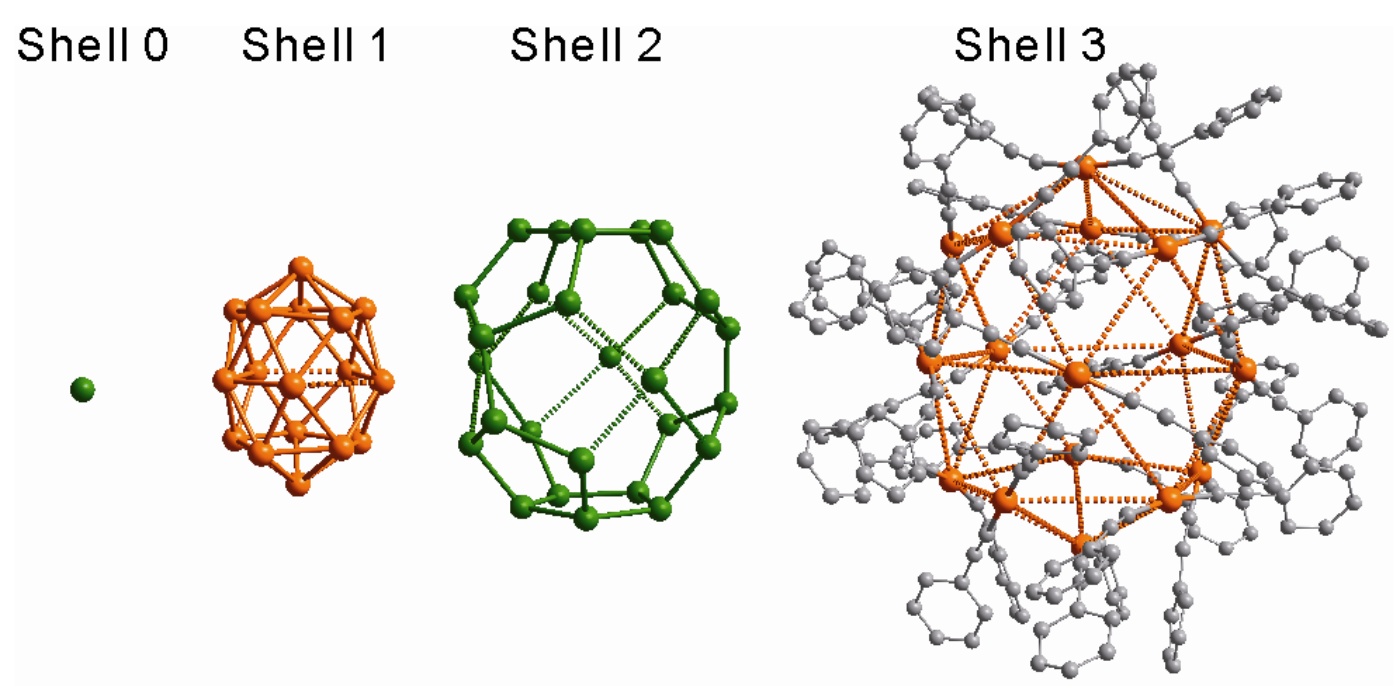

Figure S2. The structures of different shells in $\mathrm{Au}_{34} \mathrm{Ag}_{28}(\mathrm{PA})_{34}$. 


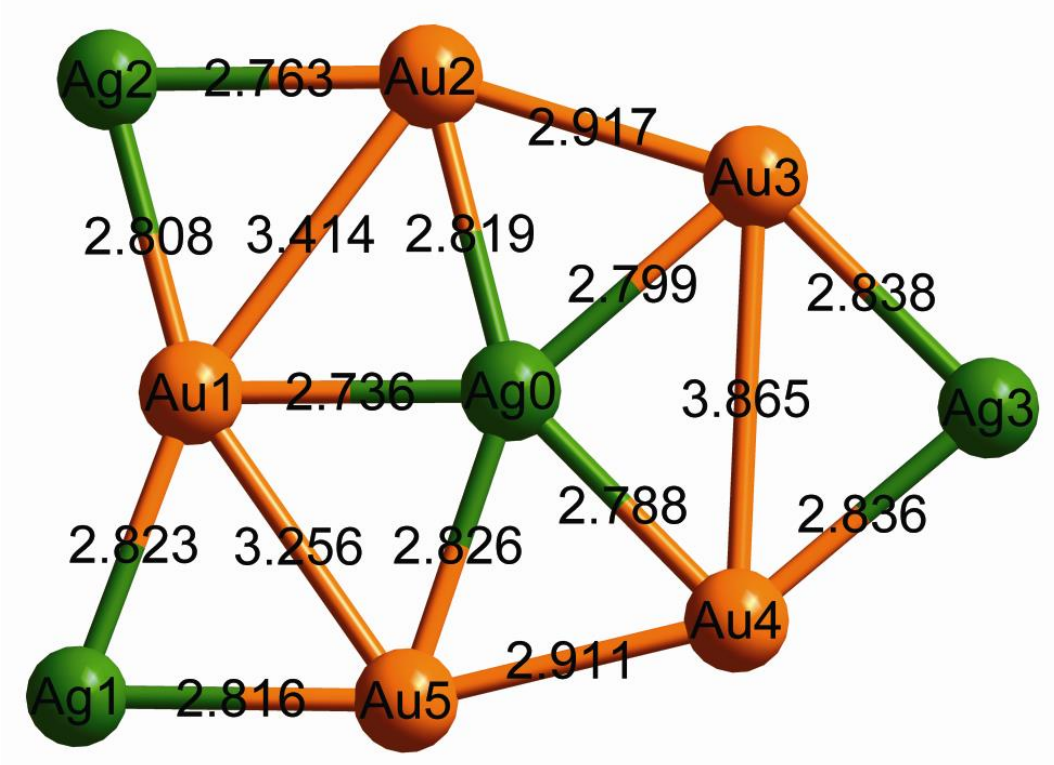

Figure S3. Top view of the distorted $\mathrm{Ag}$ centered $\mathrm{Au}_{5}$ pentagon and three surrounding $A\left(\mathrm{Ag}_{1}, \mathrm{Ag}_{2}, \mathrm{Ag}_{3}\right)$ atoms at the center for the cluster. Bond lengths are given in $(\AA)$. The three $A$ atoms $\left(\mathrm{Ag}_{1}, \mathrm{Ag}_{2}, \mathrm{Ag}_{3}\right)$ reside on the equatorial plane and are four-coordinated while the remaining four (two pairs) are off the equatorial plane and, like all the other atoms in Shell-2, are three-coordinated, to other atoms in the same shell.
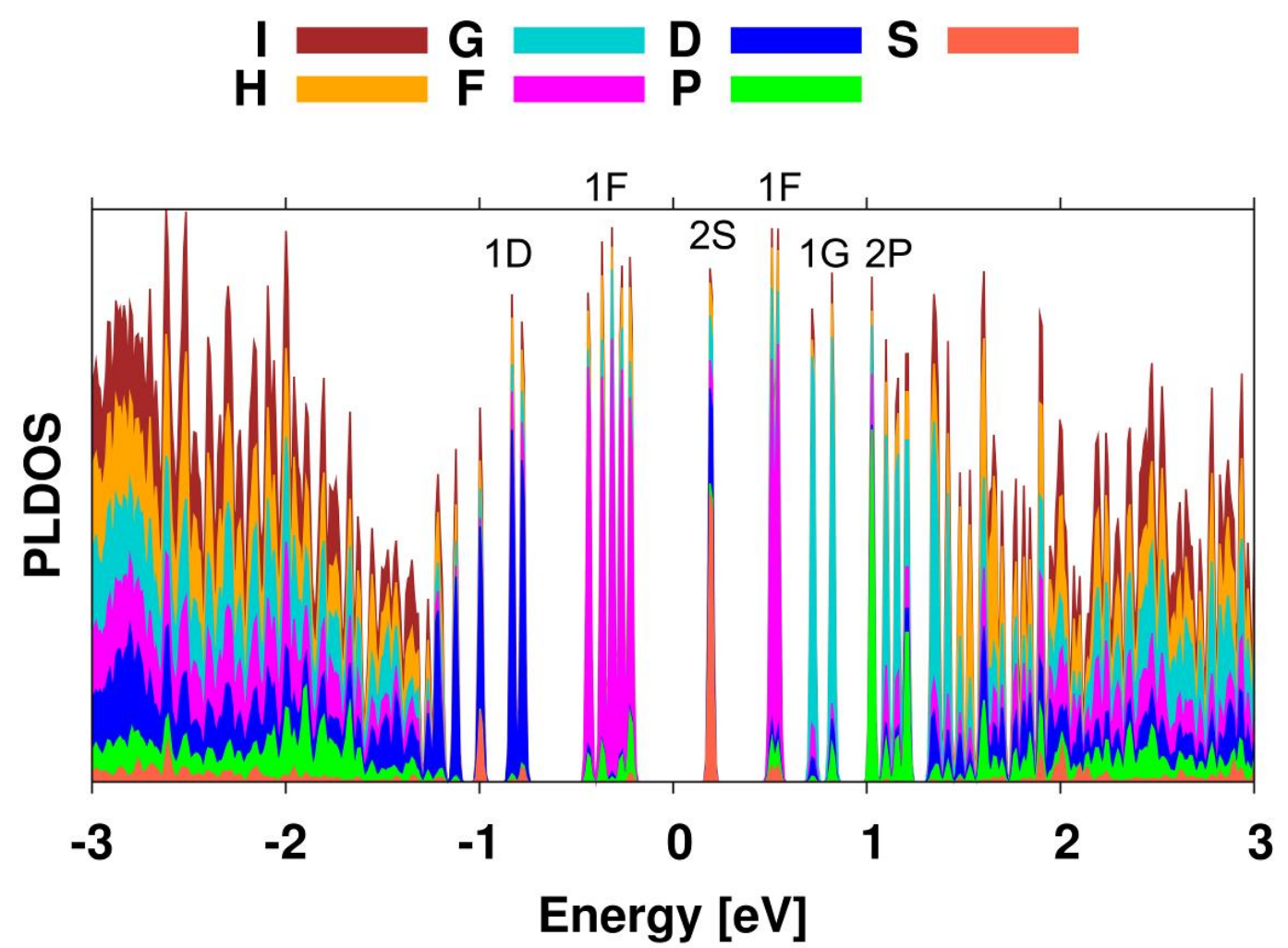

Figure S4. Density of electronic states, projected to the superatom symmetries for $(\mathrm{AuAg})_{62}(\mathrm{PA})_{34}$ cluster. The Fermi energy is at zero. 


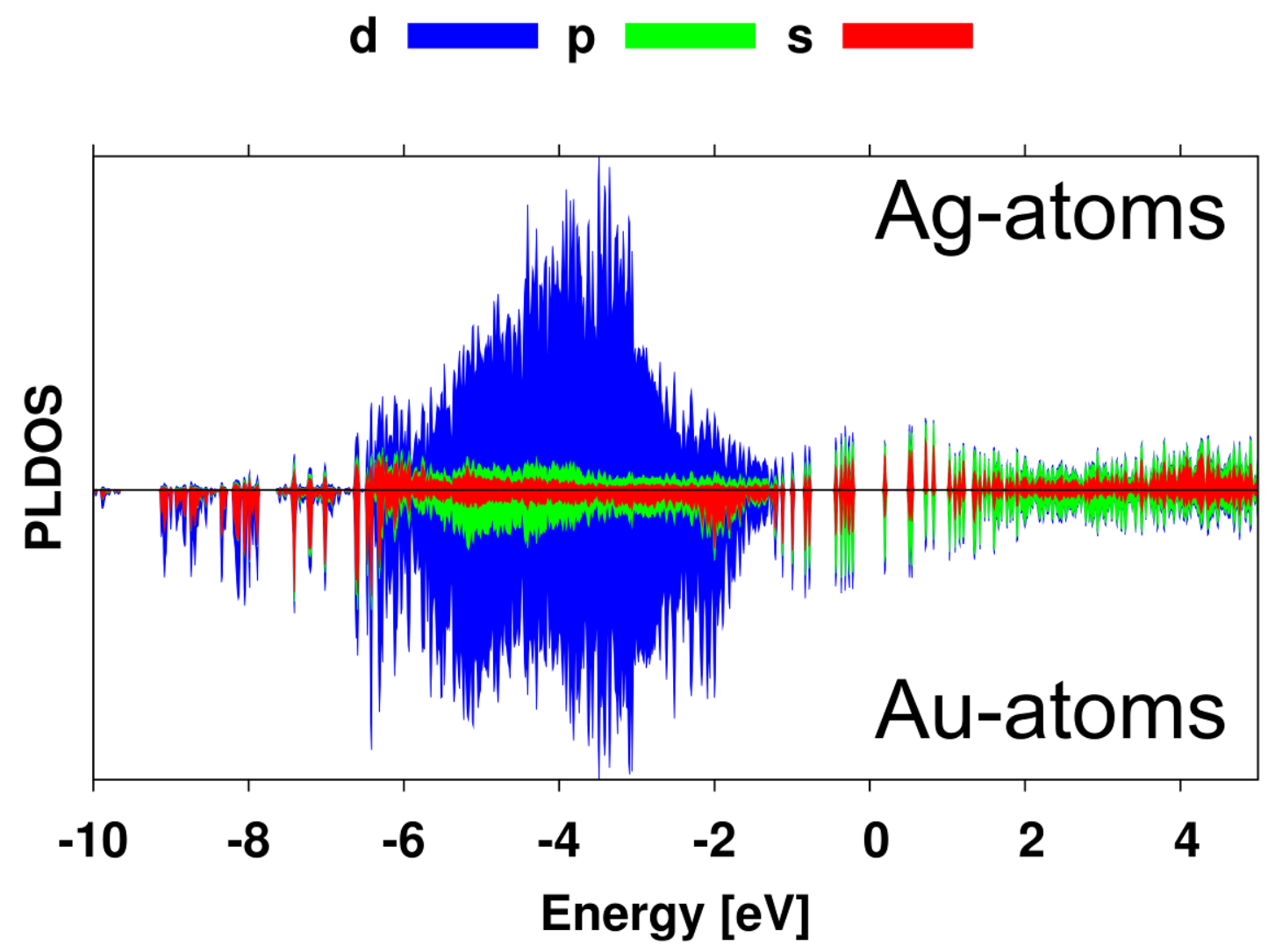

Figure S5. Decomposition of the electronic states of the $(\mathrm{AuAg})_{62}(\mathrm{PA})_{34}$ cluster into the $\operatorname{Ag}(4 d), A u(5 d), A g(5 s p)$ and $A u(6 s p)$ contributions. The Fermi energy is at zero. The d-electrons $(\mathrm{Au}(5 \mathrm{~d}))$ start to dominate at energies below $-2 \mathrm{eV}$. 
a

Energy, occ. states [eV]

PDOS
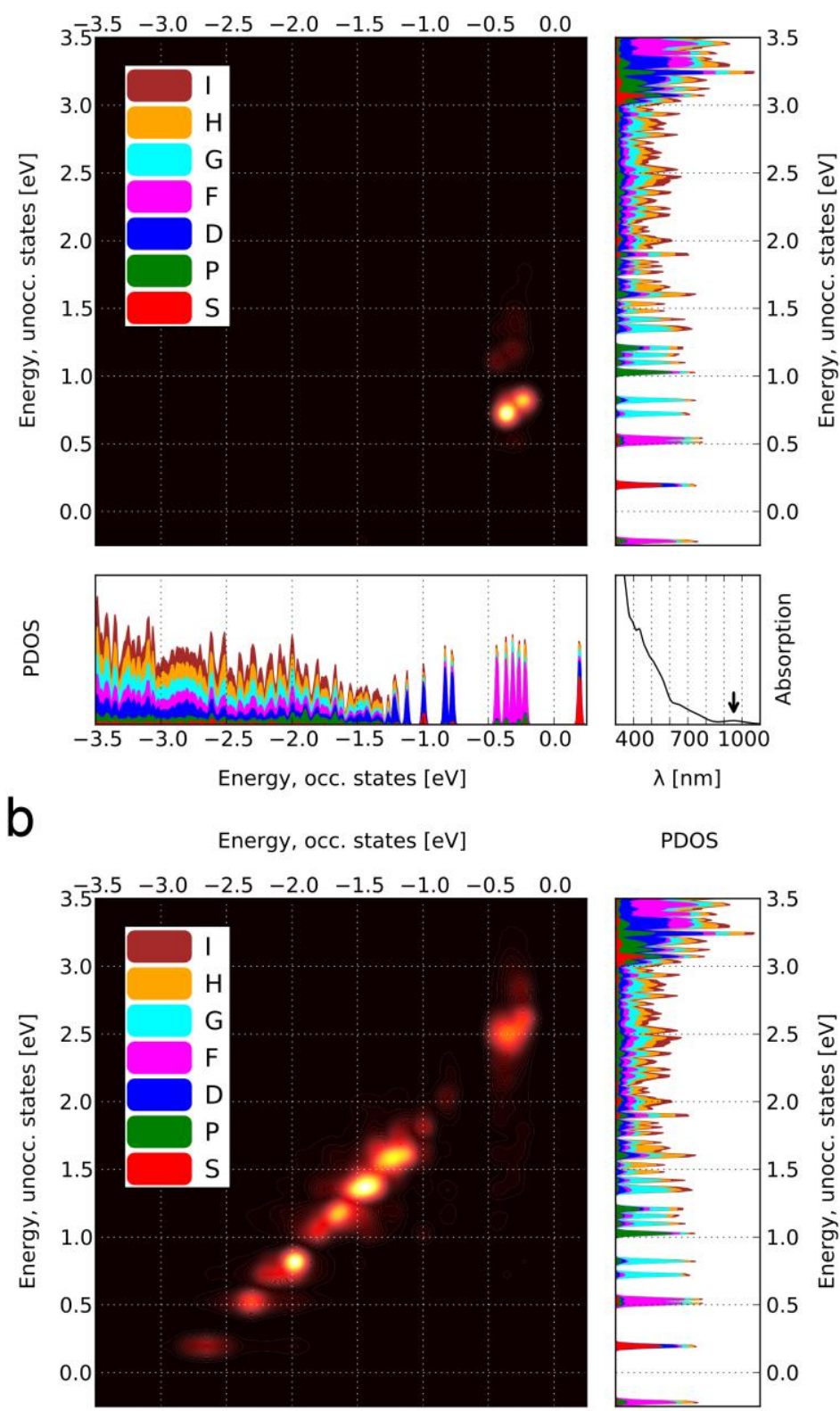

PDOS
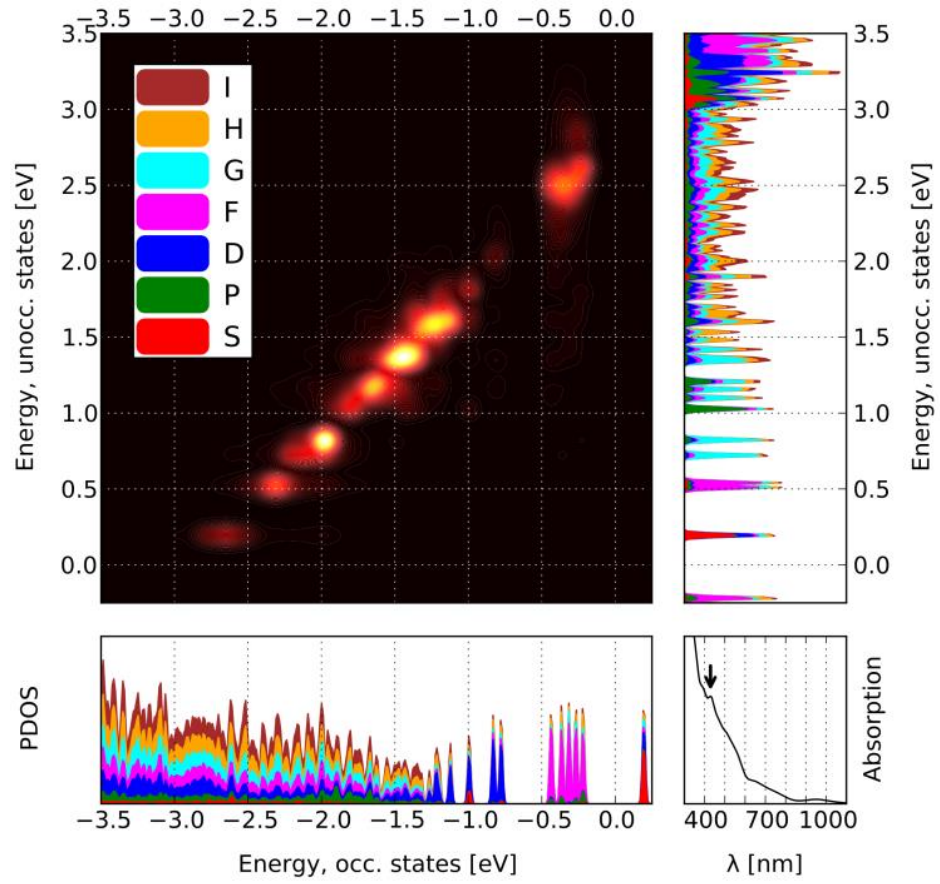

Figure S6. The transition contribution map (TCM) of absorption peaks of the $(\mathrm{AuAg})_{62}(\mathrm{PA})_{34}$ cluster at a) $951 \mathrm{~nm}(1.30 \mathrm{eV})$ and b) $429 \mathrm{~nm}(2.89 \mathrm{eV})$. The lower left and upper right panels show the density of the occupied and unoccupied states, respectively, projected to the superatom symmetries. The upper left panel shows the intensity of the individual transitions as a 2D color map from black (low contribution) to bright yellow (high contribution). The lower right panel shows the calculated absorption spectrum and the arrow points to the peak that is analyzed. 

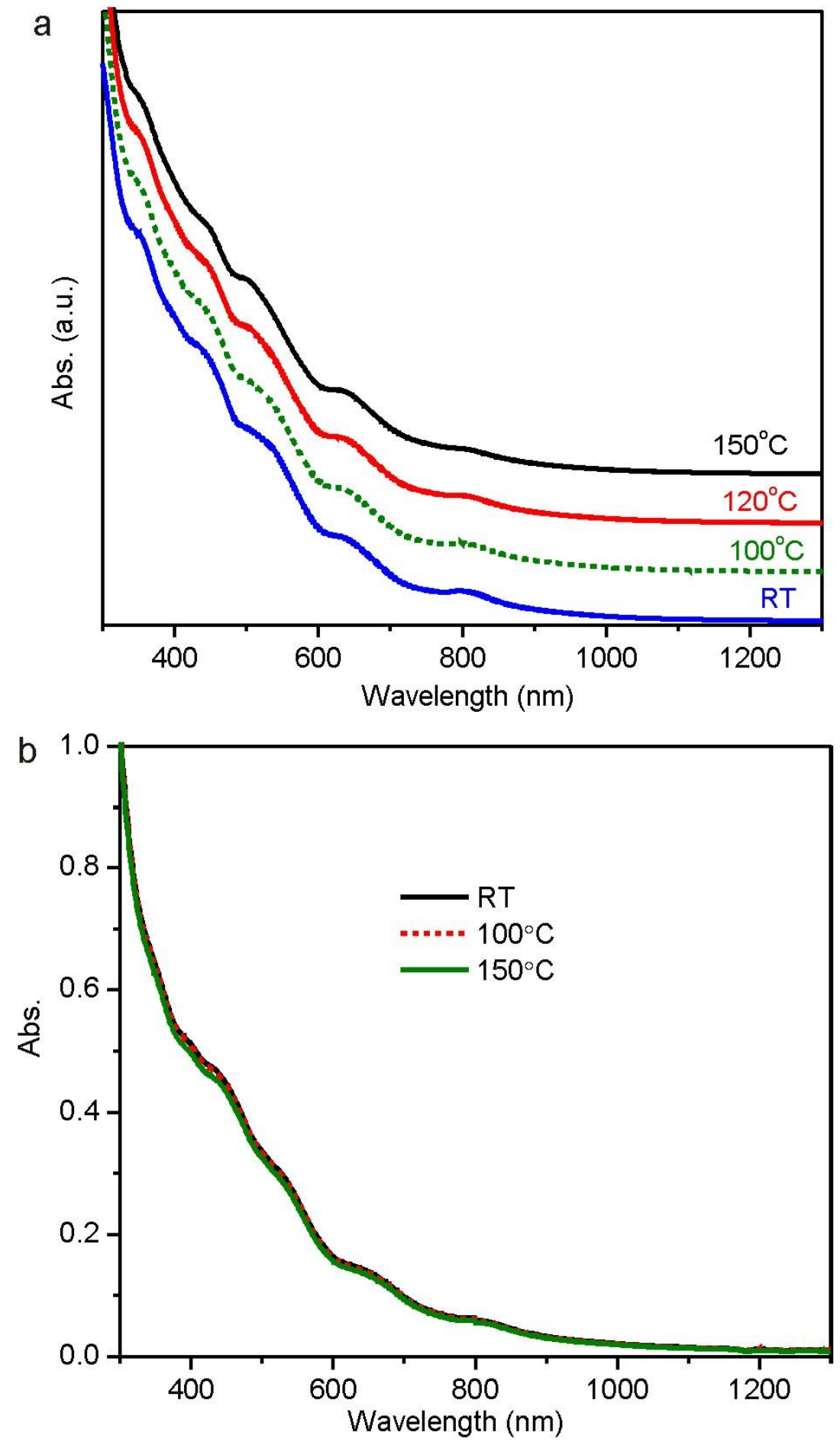

Figure S7. (a) Optical spectra of the $\mathrm{Au}_{34} \mathrm{Ag}_{28}(\mathrm{PA})_{34}$ nanocluster after being heated in the solid-state form at RT, 100,120 and $150^{\circ} \mathrm{C}$ for $1 \mathrm{~h}$, showing that the peak positions were not shifted after thermal treatments. (b) Optical spectra of three equal portions of $\mathrm{Au}_{34} \mathrm{Ag}_{28}(\mathrm{PA})_{34}$ nanoclusters after being heated in the solid-state form at RT, 100 and $150^{\circ} \mathrm{C}$ for $1 \mathrm{~h}$ and re-dissolved in the same amount of THF as the solvents, showing that the absorption intensities of the clusters after the thermal treatments were about the same. 


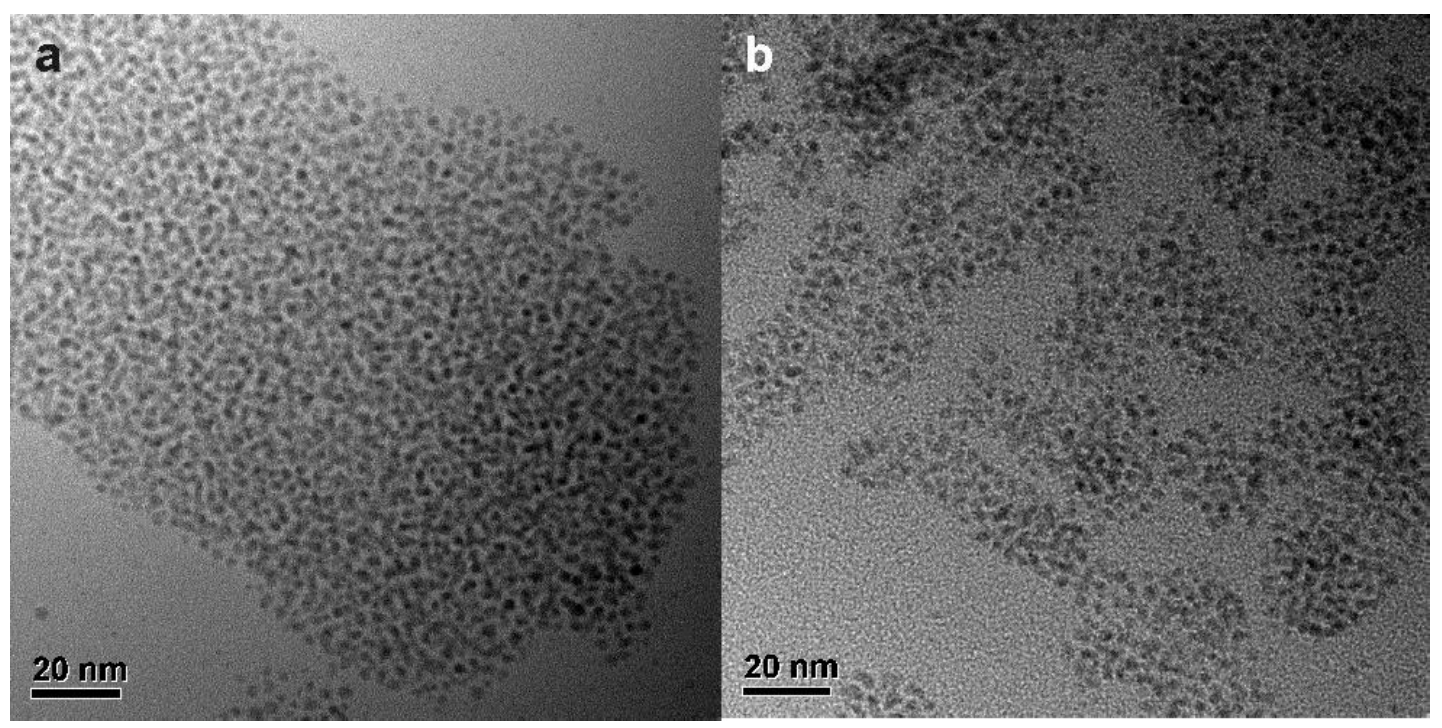

Figure S8. TEM images of the cluster before (a) and after (b) heating in the solid-state form at $150^{\circ} \mathrm{C}$ for $1 \mathrm{hr}$. The solids were re-dissolved in $\mathrm{CH}_{2} \mathrm{Cl}_{2}$ for the preparation of TEM samples.

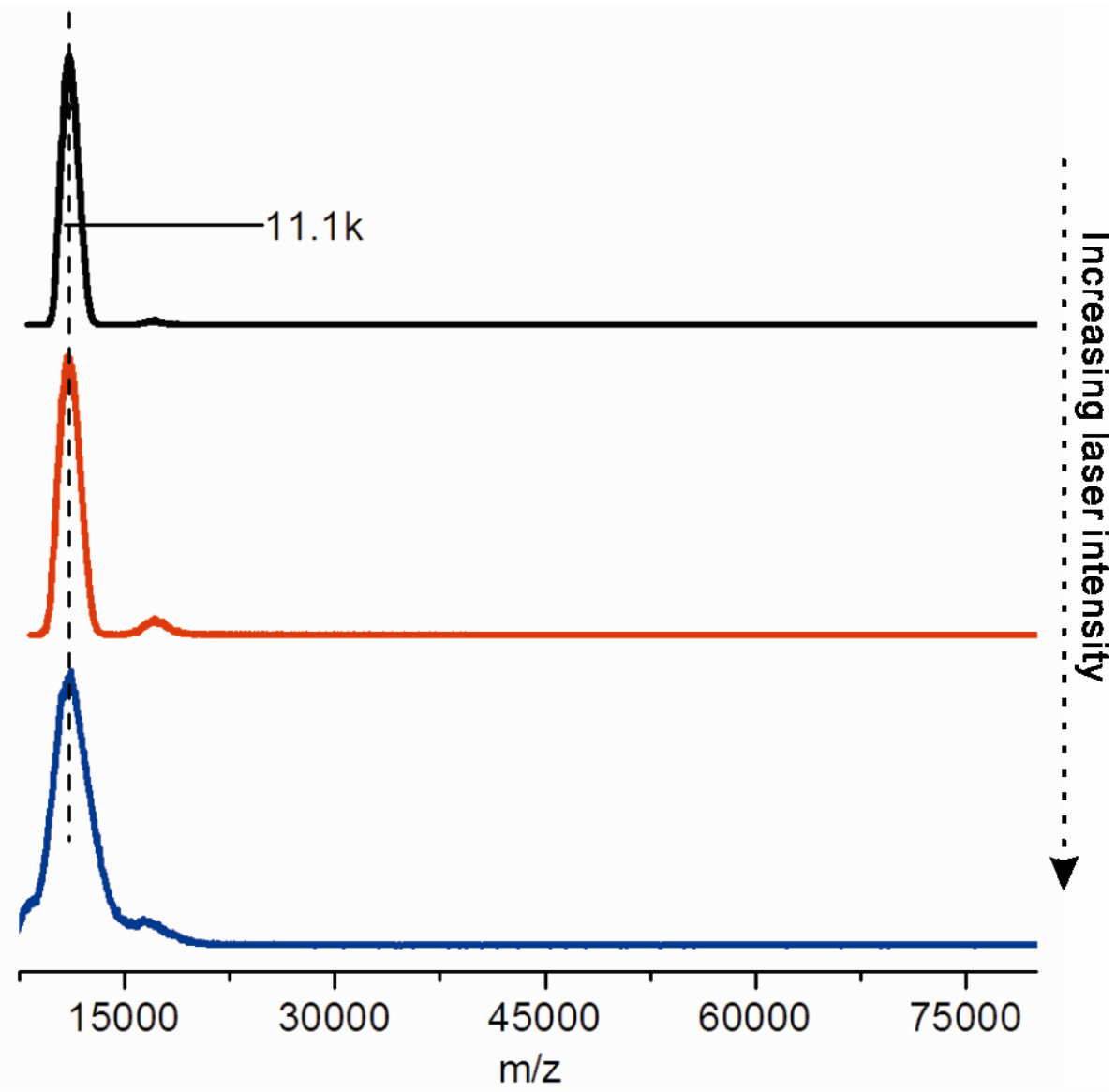

Figure S9. Positive mode MALDI-TOF mass spectrum of $\mathrm{Au}_{34} \mathrm{Ag}_{28}(\mathrm{PA})_{34}$ after thermal treatment in the solid-state form at $150^{\circ} \mathrm{C}$ for $1 \mathrm{~h}$ with laser pulse intensities increased from $20 \%, 50 \%$ to $90 \%$ ( from top to bottom panels). 

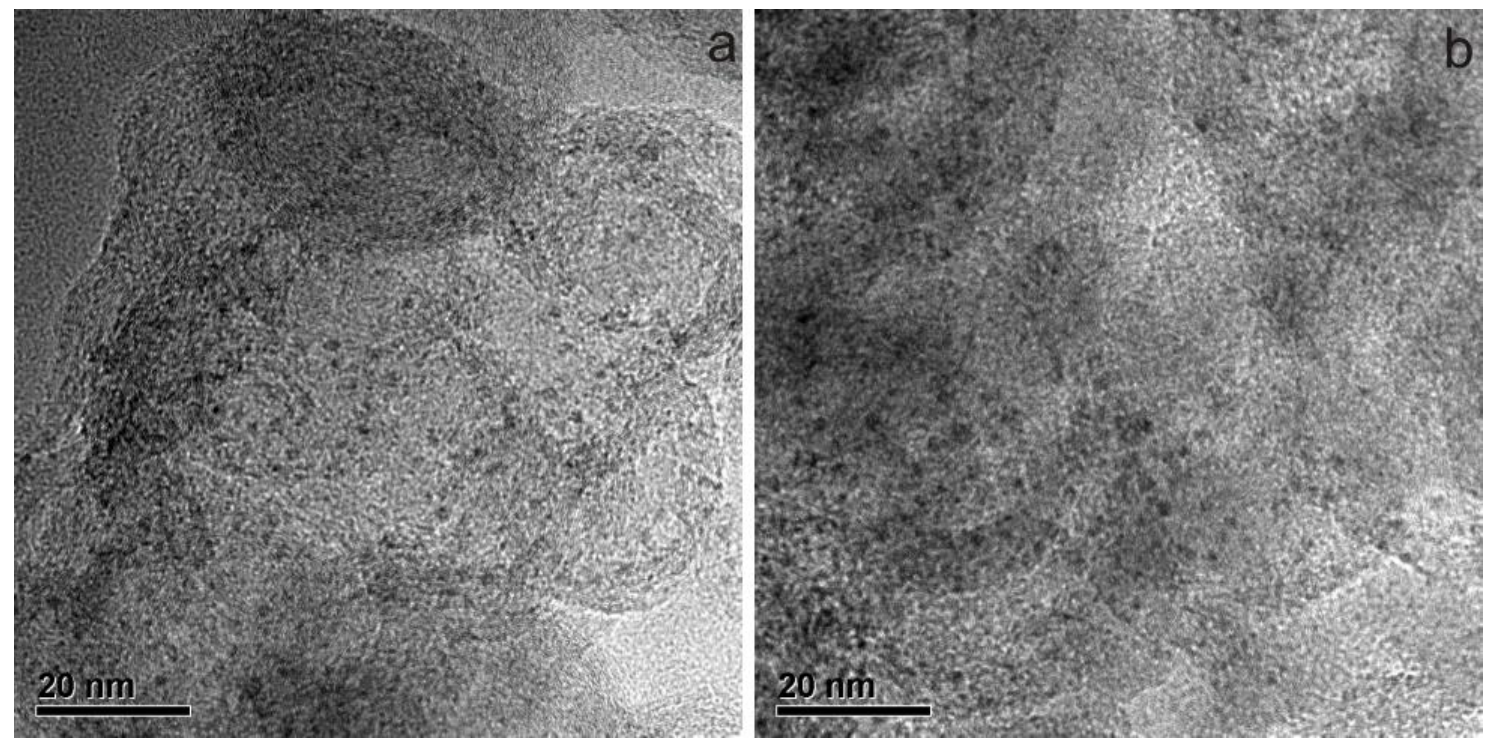

Figure S10. TEM image of (AuAg) ${ }_{62} / \mathrm{XC}-72$ before (a) and after (b) heating under vacuum at $150^{\circ} \mathrm{C}$ for $1 \mathrm{hr}$.
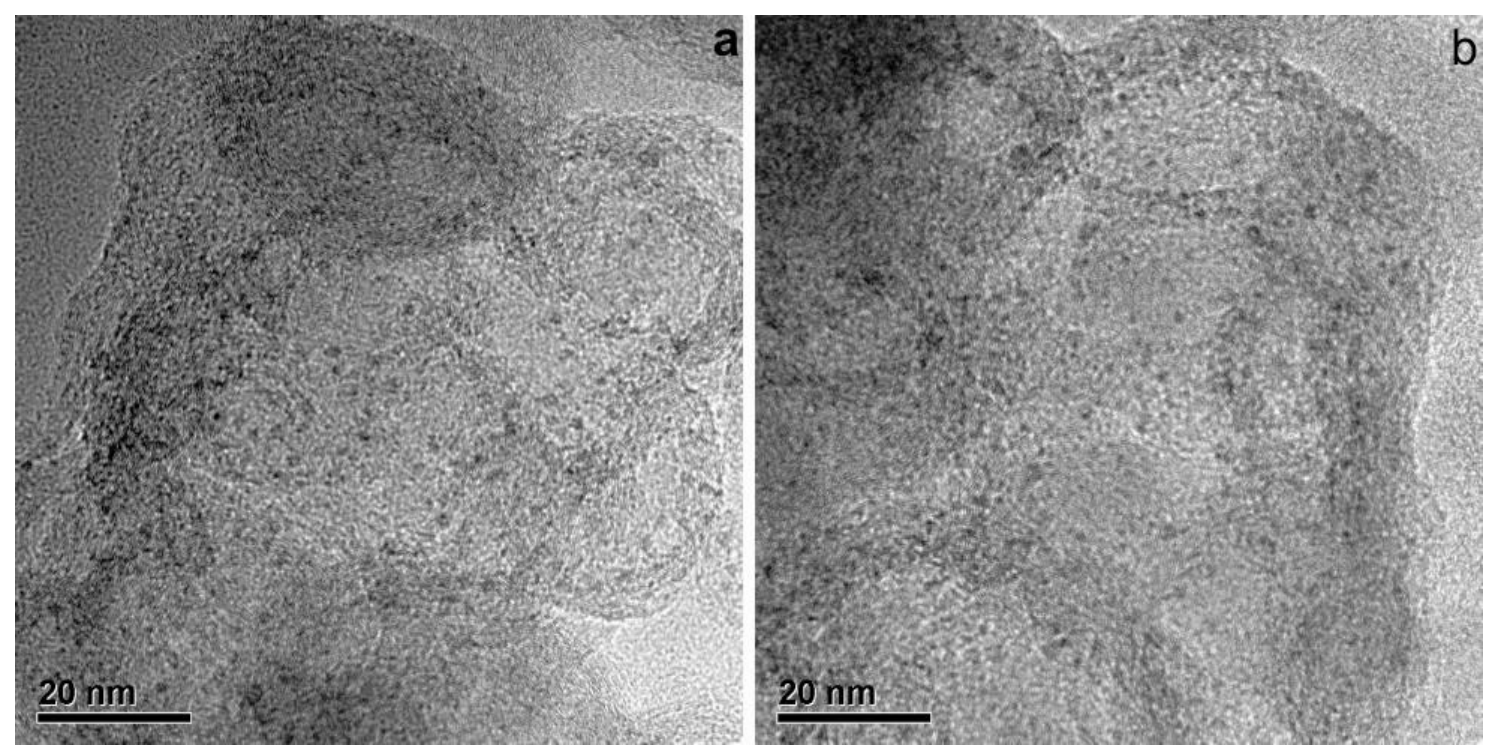

Figure S11. TEM images of the cluster before (a) and after (b) the catalytic reaction. 


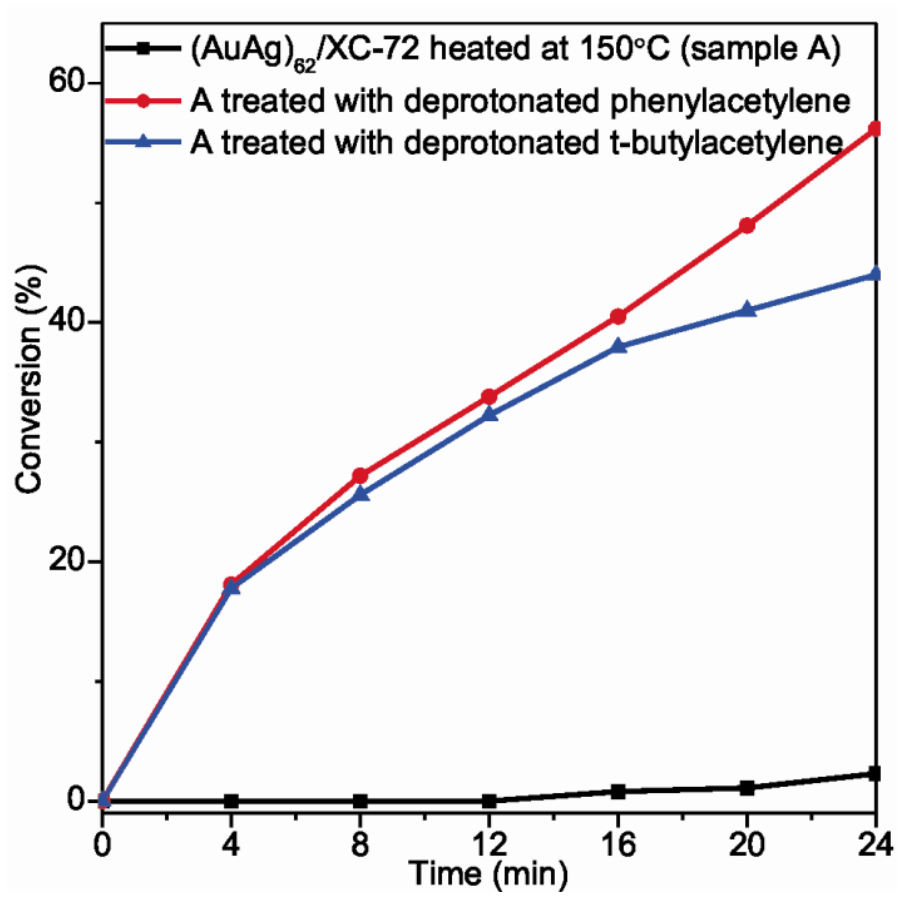

Figure S12. Catalytic performances of ligand-off cluster before and after treatment with different deprotonated terminal alkynes.
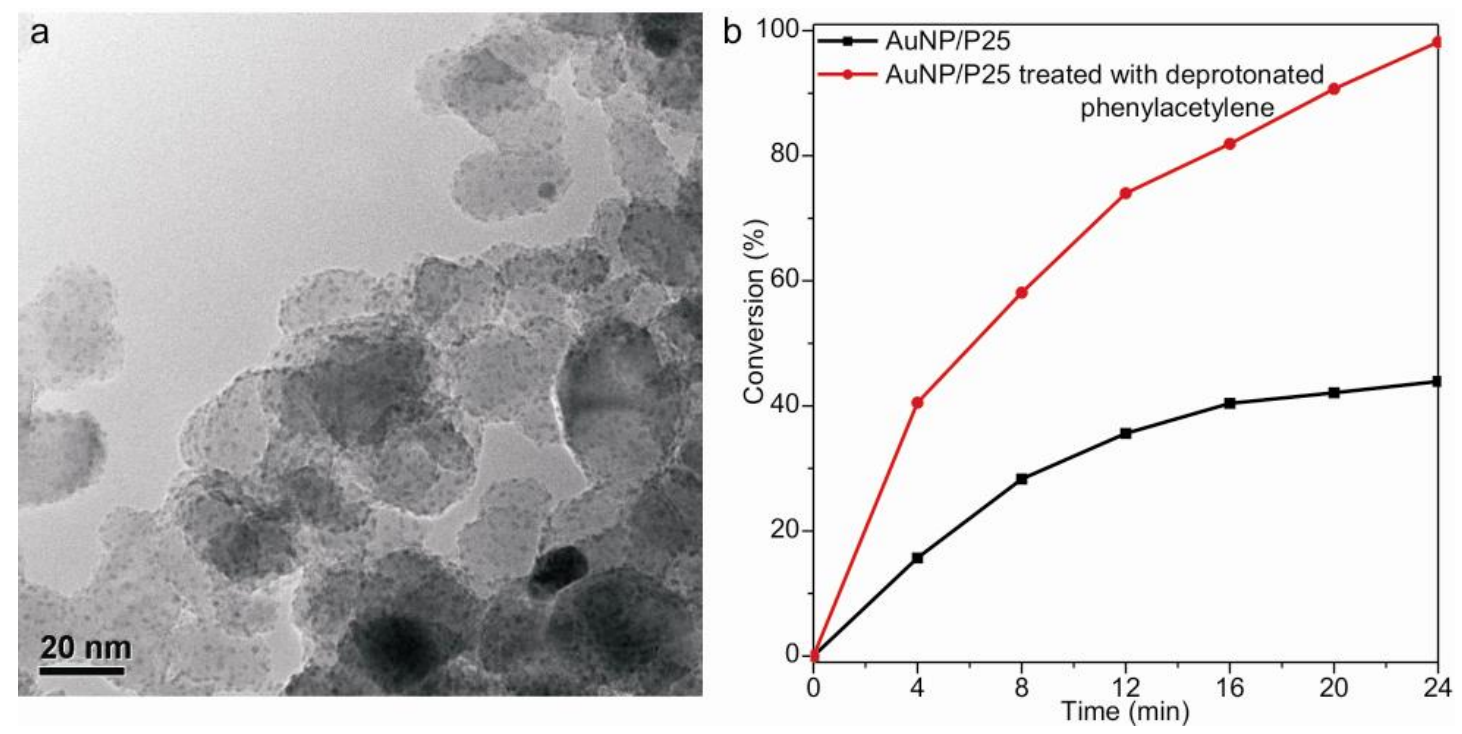

Figure S13. (a) Representative TEM image of $\mathrm{TiO}_{2}(\mathrm{P} 25)$-supported Au nanoparticles prepared by the conventional deposition-precipitation method. (b) Catalytic performances of $\mathrm{TiO}_{2}(\mathrm{P} 25)$-supported $\mathrm{Au}$ nanoparticles before and after treatment with deprotonated phenylacetylene. 


\section{References:}

(1) Nishina, N.; Yamamoto, Y. Synlett 2007, 11, 1767.

(2) Peña-López, M.; Ayán-Varela, M.; Sarandeses, L. A.; Pérez Sestelo, J. Chem. -Eur. J. 2010, 16, 9905.

(3) CrysAlis ${ }^{\text {Pro } V e r s i o n ~ 1.171 .35 .19 . ~(2011) . ~ A g i l e n t ~ T e c h n o l o g i e s ~ I n c . ~ S a n t a ~ C l a r a, ~}$ CA, USA.

(4) Sheldrick, G. M. Acta Crystallographica Section A 2008, 64, 112.

(5) Dolomanov, O. V.; Bourhis, L. J.; Gildea, R. J.; Howard, J. A. K.; Puschmann, H. Journal of Applied Crystallography 2009, 42, 339.

(6) Enkovaara, J.; Rostgaard, C.; Mortensen, J.J.; Chen, J.; Dulak, M.; Ferrighi, L.; Gavnholt, J.; Glinsvad, G.; Haikola, V.; Hansen, H.; Kristoffersen, H.; Kuisma, M.; Larsen, A.; Lehtovaara, L.; Ljungberg, M.; Lopez- Acevedo, O.; Moses, P.; Ojanen, T.; Olsen, T.; Petzold, V.; Romero, N.; Stausholm- Moller, J.; Strange, M.; Tritsaris, G.; Vanin, M.; Walter, M.; Hammer, B.; Hakkinen, H.; Madsen, G.; Nieminen, R.; Norskov, J.; Puska, M.; Rantala, T.; Schiotz, J.; Thygesen K. and Jacobsen, K.W. J. Phys.: Condens. Matter 2010, 22, 253202.

(7) Walter, M; Akola, J.; Lopez-Acevedo, O.; Jadzinsky, P.; Calero, G.; Ackerson, C.; Whetten, R.L.; Grönbeck, H.; Häkkinen, H. Proc. Natl. Acad. Sci. U.S.A. 2008, 105, 9157.

(8) Walter, W.; Häkkinen, H.; Lehtovaara, L.; Puska, M.; Enkovaara, J.; Rostgaard, C.; Mortensen, J.J. J. Chem. Phys. 2008, 128, 244101.

(9) Malola, S.; Lehtovaara, L.; Enkovaara, J.; and Häkkinen, H. ACS Nano 2013, 7, 10263.

(10) Dass, A.; Theivendran, S.; Nimmala, P. R.; Kumara, C.; Jupally, V. R.; Fortunelli, A.; Sementa, L.; Barcaro, G.; Zuo, X.; Noll, B. C. J. Am. Chem. Soc. 2015, 137, 4610.

(11) Zeng, C. J.; Chen, Y. X.; Kirschbaum, K.; Appavoo, K.; Sfeir, M. Y.; Jin, R. C. Sci. Adv. 2015, 1 , e1500045. 